\title{
Geostatistical merging of weather radar data with a sparse rain gauge network in Queensland
}

\author{
C. Sharp ${ }^{a}$, A. Schepen ${ }^{b}$, S. Das ${ }^{a}$ and Y. Everingham ${ }^{a}$ \\ ${ }^{a}$ James Cook University, ${ }^{b}$ CSIRO \\ Email: callum.sharp@my.jcu.edu.au
}

\begin{abstract}
Many parts of Australia, including much of Queensland and Northern Australia, tend to have sparse rain gauge coverage. To provide rainfall information across Australia, several gridded daily rainfall datasets such as those available through the Australian Water Availability Project and Scientific Information for Land Owners services have been developed. These daily grids are produced by interpolation of rain gauge data and therefore can provide unrealistic rainfall estimates in areas that have few rain gauges. To obtain rainfall data at a higher spatial resolution, weather radars and satellites can provide coverage over a large area although their measurements come with considerable uncertainty.
\end{abstract}

Various approaches have been developed to adjust radar and satellite data and statistically merge them with rain gauge measurements in interpolation schemes, the goal being to retain the information on the spatial distribution of rainfall provided by remote sensing while also taking advantage of the greater accuracy of the rain gauges, but many of these techniques have been applied primarily on shorter time scales of an hour or less.

This paper applies some existing methods for geostatistical merging of radar data with sparse rain gauge networks and evaluates the performance of the approaches using the Mt Stapylton radar in Brisbane and 15 surrounding rain gauges. Summer and winter data from 01/12/2013 to 28/02/2018 are considered. The radar data is corrected for mean field bias using quantile mapping and is used to develop the variogram models for use in Kriging. The performance of Kriging the gauge data using the radar variogram is compared with conditional merging and Kriging with radar values introduced as a drift variable. Leave-one-out crossvalidation is used to evaluate the performance of the methods.

We find some disagreement between all radar-based approaches and the validation gauge measurements with typical daily root-mean-square errors being between $10 \mathrm{~mm}$ and $20 \mathrm{~mm}$ for all approaches. Some outliers with substantially higher RMSE are noted for some days in the unadjusted radar data as well as in the corrected and interpolated data. For winter data the bias-correction and interpolation steps increased the agreement between the radar data and the validation gauges, but this improvement was not observed in the summer data. In addition, due to the low number of gauges the performance of the interpolation is extremely sensitive to the rain gauge values, with certain combinations of rain gauge values and choice of validation gauge leading to extremely large cross-validation errors. The results indicate that while incorporating the radar data makes it possible to perform Kriging with few gauges on a single day's data, this is not an ideal approach for quantitative precipitation estimation and further steps should be taken to improve the radar-gauge correlation.

Keywords: $\quad$ Weather radar, Kriging, rainfall, quantile mapping 


\section{INTRODUCTION}

For the purposes of agricultural modelling, hydrological modelling, water availability assessment and climate analysis it is crucial to have access to high quality precipitation data. To address the demand for precipitation data in Australia, some gridded rainfall products have been developed based on rainfall data retrieved from rain gauges. Two commonly used products are the AWAP (Australian Water Availability Project) and SILO (Scientific Information for Land Owners) daily gridded rainfall datasets. The AWAP grids are derived from smoothing spline interpolation of gauge data as described in Jones et al. (2009) while SILO grids are generated from a combination of spline interpolation and ordinary kriging of rain gauge data shown in Jeffrey et al. (2001).

Many areas of Australia including much of Queensland and Northern Australia contain regions which have low spatial density of rain gauges. It can be challenging to make accurate interpolated rainfall grids in these regions since rain events that are smaller than the typical distance between rain gauges may not be adequately sampled by the gauges. In these regions, remote sensing techniques such as satellite observation and weather radars can provide extra spatial detail. Many areas of Australia that contain few rain gauges are within range of one or more weather radars maintained by the Australian Bureau of Meteorology (BOM). Since BOM releases data from their extensive radar network to the public for free this provides an attractive source of highresolution rainfall data.

Weather radars do not measure rain rates directly, instead they measure reflectivity. As demonstrated by Marshall et al. (1947) for a given rain drop-size distribution (DSD) the rain rate can be estimated from radar reflectivity using a power law conversion relationship. In general, the DSDs of rain events are unknown so the correct conversion relationship for each radar measurement is also unknown. The error associated with the choice of conversion relationship is often assumed to be a uniform, multiplicative bias across the whole radar field. Chumchean et al. (2006) compares two calibration techniques for reducing the mean field bias of radar data, multiplying across the radar field by the mean gauge/radar measurement ratio and Kalman filtering. The Bureau of Meteorology provides some radar-derived rainfall accumulation data for a limited number of radars in their network and the rainfall fields for these products are calibrated with rain gauges using Kalman filtering. Rabiei and Haberlandt (2015) use quantile mapping to reduce the mean field bias of radar measurements. Calibrating remotely sensed rainfall data using rain gauges allows for the preservation of the spatial detail provided by satellite or radar while rescaling the values based on the statistical properties of the more accurate rain gauge measurements.

Ochoa-Rodriguez et al. (2019) provides a review of multiple statistical merging techniques for radar and rain gauge data in the context of urban hydrology, including Kriging with external drift (also known as universal Kriging) and conditional merging. These statistical interpolation techniques allow for the incorporation of radar measurements in the interpolation of rain gauge data. For sparse rain gauge networks, some modifications to standard merging approaches must be made such as those used in Seck and Baelen (2018) to generate rainfall estimates.

This paper applies some geostatistical merging techniques to create daily rainfall grids using a weather radar and a sparse rain gauge network in Queensland. Convective and stratiform rain events have different drop-size distributions, leading to different relationships between radar reflectivity and rain rate as described by Chumchean et al. (2008). To reduce the impact of this variation, the results of these different approaches are compared for summer and winter rainfall distributions separately as it is assumed the ratio of convective to stratiform events will vary by season.

\section{DATA}

Both the radar and rain gauge data used are obtained from BOM. The radar volumes are made available to the public through a web archive provided by Soderholm et al. (2019). These data are structured in accordance with the EUMETNET OPERA weather radar information model described by Michelson et al. (2014). We use data from the Mt Stapylton radar in Brisbane (radar ID number: 66) and from 15 rain gauges within a $150 \mathrm{~km}$ range of the radar from 01/12/2013 to 28/02/2018. Digital elevation data was sourced from Amazon Web Services' terrain tiles data set. The Brisbane radar data is supplied in polar coordinates with an azimuthal resolution of $1^{\circ}$ and a radial resolution of $250 \mathrm{~m}$. The Brisbane radar provides 1 image every 6 minutes, and each image includes up to 14 scans taken at different elevation angles ranging from $0.5^{\circ}$ to $32^{\circ}$ above the horizon. 


\section{METHODS}

We convert the 3D radar volumes to 2D pseudo-Constant Altitude Plan Position Indicator (pCAPPI) grids with a resolution of $2.5 \mathrm{~km}$ by $2.5 \mathrm{~km}$. To avoid ground clutter, an altitude of $1.5 \mathrm{~km}$ was chosen for the pCAPPIs. The values on the pCAPPI grid were calculated by averaging all overlapping radar measurements from the nearest elevation angle whose beams pass over (or under) the grid point. Once they were calculated, pCAPPI grids were treated as ground-level measurements and the $\mathrm{z}$-values are replaced with the surface terrain elevation under the grid location retrieved from the Amazon Web Services' terrain tiles. A zoom level of 6 is chosen for the terrain tiles as it corresponds to a resolution close to that of the pCAPPI grid. The performance of the bias correction and interpolation schemes in the remainder of this section will be assessed using leave-one-out crossvalidation comparisons with the rain gauges.

\subsection{Calculating daily radar-derived rainfall grids}

Radar reflectivity measurements can be converted to rain rates that are used to calculate daily rainfall totals. The reflectivity factor, $Z$, is related to the rain rate, $R$ (in $\mathrm{mm} / \mathrm{h}$ ) by the $\mathrm{Z}-\mathrm{R}$ relation

$$
Z=a R^{b}
$$

and we use the "default" Z-R relationship parameters, known as the Marshall-Palmer relationship, where $a=$ 200 and $b=1.6$. Next, for each day of interest in the data sets, rainfall totals are calculated from the radar rain rates using the trapezoidal rule

$$
R_{\text {Radar }}\left(x, y, z, t_{d}\right)=\left(\frac{1}{a}\right)^{\frac{1}{b}} \sum_{n} \frac{Z\left(x, y, z, t_{n-1}\right)^{\frac{1}{b}}+Z\left(x, y, z, t_{n}\right)^{\frac{1}{b}}}{2} \Delta t_{n}
$$

where $t_{d}$ is the day and $\Delta t_{n}$ is the time interval (in hours) between the $n-1$ th and $n$th radar scans.

\subsection{Correcting for mean field bias}

The approach chosen for bias correction is known as quantile mapping. We fit a theoretical distribution to the empirical cumulative probability density function (CDF) of the radar and rain gauge measurements. The gamma distribution is commonly fitted to precipitation data and the mixed Bernoulli-gamma distribution is widely used for precipitation data containing zeroes as described by Thom (1968). Since our data contains many zeroes, the mixed distribution is chosen. Once the theoretical CDFs are fitted, the quantiles of the radar rainfall distribution are converted to their equivalent corresponding rainfall values for the rain gauge distribution. The quantile mapping step can be expressed as

$$
R_{\text {Radar }}^{\prime}\left(x, y, t_{d}\right)=F_{\text {Gauge }}^{-1}\left(F_{\text {Radar }}\left(R_{\text {Radar }}\left(x, y, t_{d}\right)\right)\right)
$$

where $F_{\text {Radar }}$ and $F_{\text {Gauge }}$ refer to the fitted CDFs of the radar and rain gauge distributions respectively. For the Bernoulli-gamma distribution the CDFs are given by

$$
F(x)=\left\{\begin{array}{c}
1-\pi+\frac{\pi}{\Gamma(k)} \gamma\left(k, \frac{x}{\theta}\right), \quad \text { if } x>0 \\
1-\pi, \quad \text { if } x \leq 0
\end{array}\right.
$$

where the distribution parameters $k$ and $\theta$ are fitted based on the empirical distribution quantiles. Quantile mapping is performed on summer and winter data independently.

\subsection{Interpolation of Rain Gauge Data}

The adjusted radar rainfall fields generated in 3.2 are used to interpolate the rain gauge data onto the radar's spatial grid using three different approaches. The approaches tested are universal Kriging both with and without radar data as a drift variable as well as conditional merging (CM). All three of these approaches involve Kriging. The Kriging equation is

$$
\hat{R}\left(\widetilde{r_{0}}\right)=\sum_{p=1}^{P} \mathrm{w}_{p} R\left(\widetilde{r_{p}}\right)
$$

where $w_{p}$ are the Kriging weights. Due to the presence of drift variables in the Kriging system, the expected precipitation value is not constant within the interpolation area. The expected value is linearly dependent on a set of background variables $Y$ given by 


$$
E\left[\hat{R}\left(\tilde{r}_{0}\right) \mid Y\left(\tilde{r}_{0}\right)\right]=a+\sum_{l=1}^{k} b_{l} Y_{l}\left(\tilde{r}_{0}\right)
$$

and the Kriging weights are calculated subject to the following conditions

$$
\begin{gathered}
\sum_{p=1}^{P} w_{p} Y_{l}\left(\tilde{r}_{p}\right)=Y_{l}\left(\tilde{r}_{0}\right) \\
\sum_{p=1}^{P} w_{p}=1 \\
\sum_{p=1}^{P} w_{p} C\left(\widetilde{r_{a}}-\widetilde{r_{p}}\right)+\sum_{l=1}^{k} \mu_{l} Y_{l}\left(\tilde{r}_{a}\right)=C\left(\widetilde{r_{a}}-\widetilde{r_{0}}\right), a=1,2, \ldots P
\end{gathered}
$$

where $C(\tilde{h})$ is the covariance function for distance vector $\tilde{h} . \widetilde{r_{p}}$ and $\widetilde{r_{a}}$ are rain gauge locations and $\widetilde{r_{0}}$ is the point being interpolated. The value $\mu_{l}$ is the Lagrange multiplier for the $l$ th unbiased condition. To perform Kriging, the covariance function must be estimated from the empirical variogram of the data. We estimate covariance function using two common parametric variogram models, exponential and spherical, as described by Cressie (1993). The model chosen for use in Kriging for a given day was based on which of the two minimise the sum of squares error between the model and experimental variograms for the day's radar rainfall field.

Rain gauges are interpolated onto the radar grid using spatial coordinates as regressors with the linear model and residual variogram model trained on the radar data. We consider the performance of using radar data as a regressor but estimating the parameter for the radar data is difficult due to the low number of rain gauges. To use the radar data as a regressor, we apply a similar method to Seck and Baelen (2018). First the rain gauge data are interpolated onto the radar grid using universal Kriging with spatial regressors only, then the radar measurements which overlap with the rain gauges are selected and interpolated onto the radar grid using universal Kriging. A deviation grid between these two different results is calculated and a new variogram model is built on the grid of deviations. Then, Kriging is used to interpolate the rain gauge data using this new variogram with the radar data included as a drift variable. The key difference between this and conditional merging is that the radar grid is never explicitly incorporated as a regressor in the CM approach.

Conditional merging aims to estimate the Kriging error at each radar grid location and adjust the interpolated rain gauge values to account for this error. First the rain gauge data are interpolated onto the radar grid using universal Kriging with spatial variables as regressors. Second, the radar data at the rain gauge locations are selected and used to interpolate over the radar grid in the same manner. The differences between the Kriged radar data and the observed radar data at each interpolation site is calculated to obtain a grid of deviations. The deviations are added to the interpolated rain gauge grid to correct for Kriging error.

\section{RESULTS AND DISCUSSION}

The cross-validated daily root-mean-square error summaries displayed in Table 1 as well as Figure 1 and 2 show that the quality of the calculated rainfall grid is extremely sensitive to the gauge measurements. Depending on the choice of validation gauge the rainfall grid can contain extreme outliers such as those observed in the maximum RMSE recorded in Table 1 for the CM approach in Summer. Large outliers are also present in the unadjusted radar data. An inspection of some of the data on some days with unusually high RMSEs reveal the unadjusted radar data was overestimating the rainfall at the gauge by a large margin. This could indicate the presence of hail or snow, or that the drop-size distribution of the rain event differed greatly from the average DSD for which the Marshall-Palmer relation is appropriate. Even when extreme outliers are excluded as was done to produce Figures 1 and 2, rainfall grids on many days show substantial disagreement with the cross-validation gauge.

For the summer data plotted in Figure 1, the bias correction step did not increase the agreement between the radar and the validation gauge indicating that the assumption of a stationary seasonal rainfall distribution was not adequate to model the summer rainfall. None of the interpolation schemes demonstrate improved performance over simply using the unadjusted radar data in summer. Figure 2 shows that for the winter data both the bias corrected data and interpolated data have slightly higher agreement with the validation gauge than the unadjusted radar data. A potential explanation for this is that if the winter rainfall distribution was more 
spatially and temporally uniform than the summer distribution, greater agreement with the validation gauge would be obtained via quantile mapping.

Figure 3 includes examples of the interpolated rainfall grids on an example rainy day (11/12/2014) with the corresponding Kriging variance maps presented in Figure 4. Figure 5 shows the measurements reported by the rain gauges located on the grids as well as the estimates from the radar-based methods for comparison. Universal Kriging with radar drift (Figure $3(\mathrm{~d})$ ) and conditional merging (Figure 3 (e)) approaches retain spatial detail present in the radar data, meaning that these methods can be used to obtain a more "weather-like" rainfall grid than univariate approaches such as ordinary Kriging of gauge data. Performing Kriging on gauges without using radar data as a covariate result in less visible structure in the interpolated grid (Figure 3 (c)), but Table 1 shows that despite the more detailed structure the actual precipitation estimates made using CM and UK with radar drift have similar accuracy to the gauge-only approach. An inspection of the day's SILO data (Figure 3 (f)) shows that SILO estimates less rainfall to the northwest of the gauges than the calibrated radar approaches. SILO interpolation is based on rain gauge measurements, so it is possible that SILO underestimates the rainfall event's intensity some areas as much of the structure was not sampled by the rain gauges. Without gauge measurements in these areas however it is difficult to meaningfully compare the accuracy.

Table 1. Summary of the daily cross-validation root-mean-square errors (RMSE) for each treatment and season rounded to one decimal place.

\begin{tabular}{|c|c|c|c|c|c|}
\hline & $\begin{array}{l}\text { Unadjusted } \\
\text { Radar Data }\end{array}$ & $\begin{array}{c}\text { Bias } \\
\text { Correction } \\
\text { Only }\end{array}$ & $\begin{array}{c}\text { Universal Kriging } \\
\text { with no Radar } \\
\text { Drift }\end{array}$ & $\begin{array}{c}\text { Universal } \\
\text { Kriging with } \\
\text { Radar Drift }\end{array}$ & $\begin{array}{l}\text { Conditional } \\
\text { Merging }\end{array}$ \\
\hline \multirow{2}{*}{$\begin{array}{c}\text { First Quartile of Daily } \\
\text { RMSE (mm) }\end{array}$} & Summer: 0.3 & Summer: 0.2 & Summer: 0.7 & Summer: 0.6 & Summer: 0.9 \\
\hline & Winter: 0.1 & Winter: 0.1 & Winter: 0.1 & Winter: 0.1 & Winter: 0.2 \\
\hline \multirow{2}{*}{$\begin{array}{c}\text { Median Daily RMSE } \\
\text { (mm) }\end{array}$} & Summer: 1.1 & Summer: 1.4 & Summer: 2.9 & Summer: 2.4 & Summer: 2.9 \\
\hline & Winter: 0.3 & Winter: 0.3 & Winter: 0.6 & Winter: 0.8 & Winter: 0.9 \\
\hline \multirow{2}{*}{$\begin{array}{c}\text { Third Quartile of Daily } \\
\text { RMSE (mm) }\end{array}$} & Summer: 4.4 & Summer: 5.8 & Summer: 7.6 & Summer: 6.8 & \multirow{2}{*}{$\begin{array}{l}\text { Summer: } 7.8 \\
\text { Winter: } 2.4\end{array}$} \\
\hline & Winter: 1.6 & Winter: 1.9 & Winter: 2.5 & Winter: 2.5 & \\
\hline \multirow[t]{2}{*}{ Max Daily RMSE (mm) } & Summer: 99.3 & Summer: 69.0 & Summer: 87.3 & Summer: 135.6 & Summer: 681.8 \\
\hline & Winter: 87.0 & Winter: 70.4 & Winter: 65.1 & Winter: 53.4 & Winter: 46.5 \\
\hline
\end{tabular}

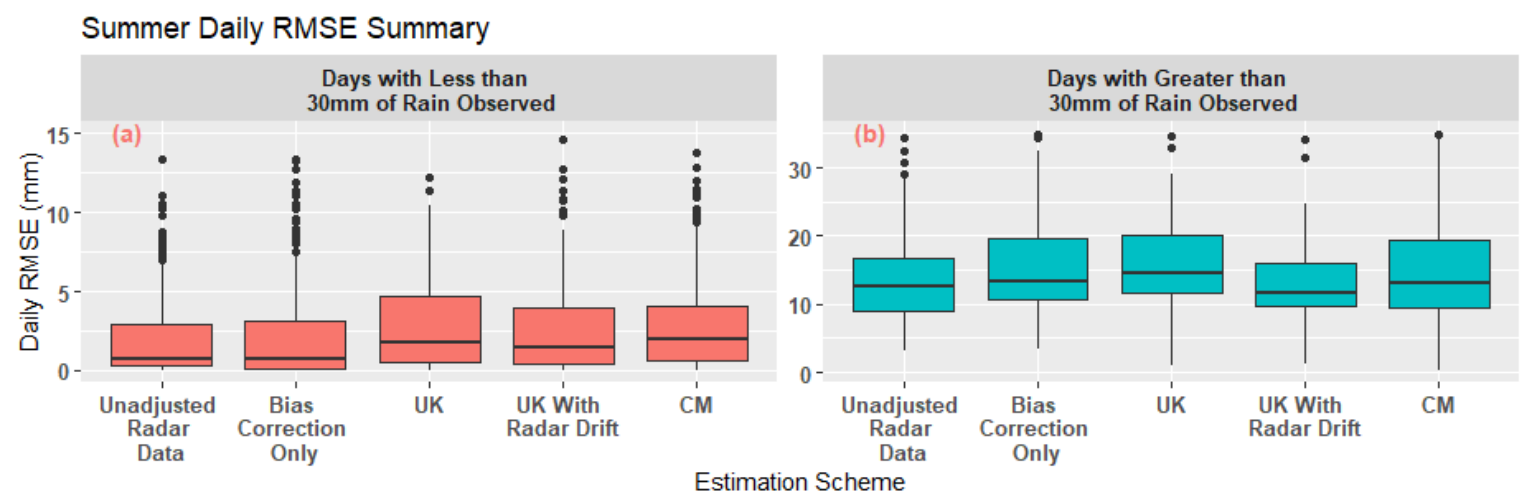

Figure 1. Boxplots of daily root-mean-square errors for summer, days where greater than $30 \mathrm{~mm}$ of rain is observed by at least one gauge are plotted separately (b). Except for the unadjusted radar data RMSEs, the RMSEs are calculated using leave-one-out cross-validation. 


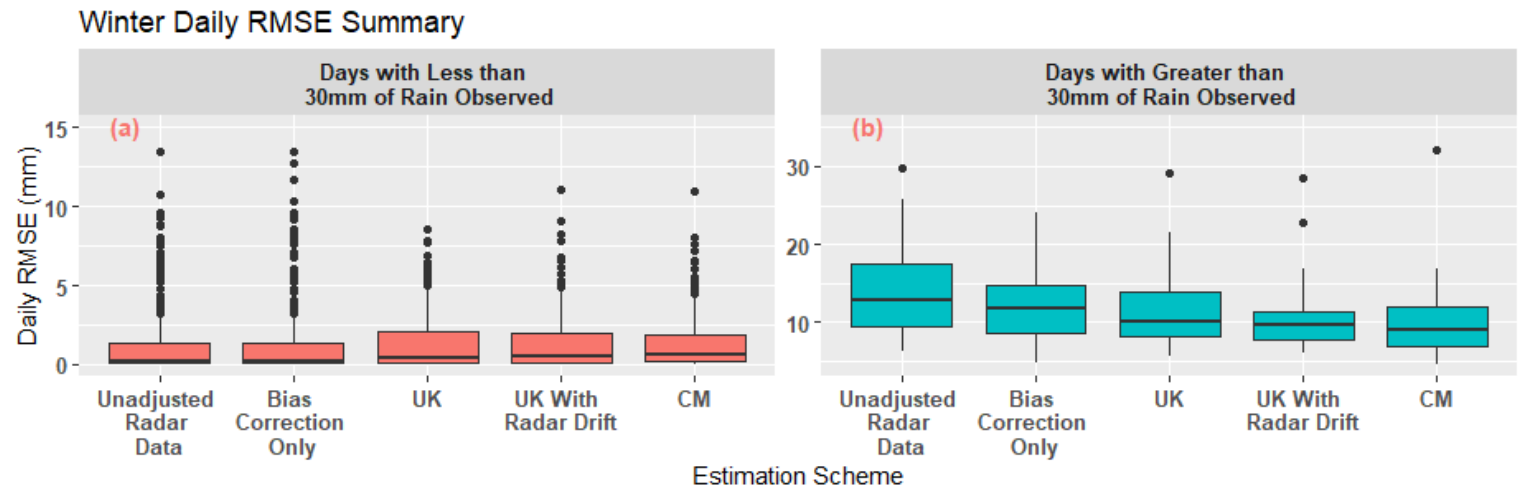

Figure 2. Boxplots of daily root-mean-square errors for winter days calculated the same way as the summer RMSEs in Figure 1.

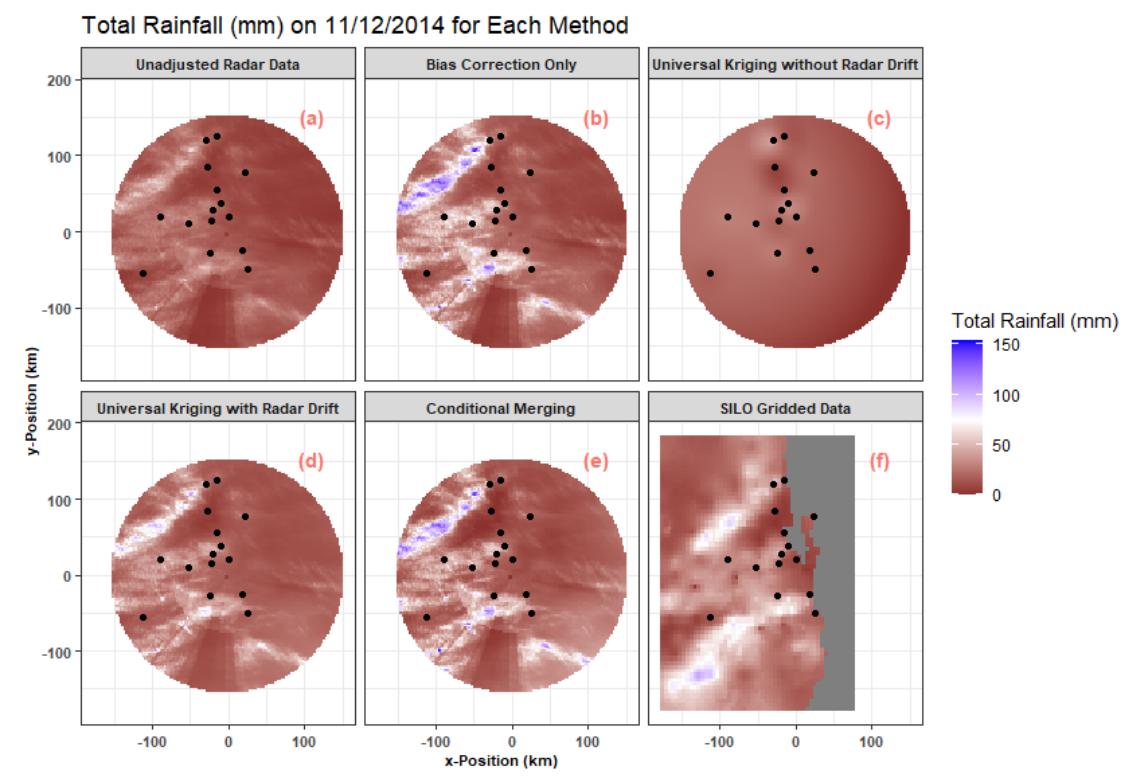

Figure 3. Plots of total daily rainfall for 11/12/2014. Included are plots for the unadjusted radar data (a), biascorrected radar data (b), UK without radar drift (c), UK with radar drift (d) and conditional merging (e) results. SILO gridded data (f) around the Brisbane radar is also presented for comparison. The rain gauge locations are marked with black points on the grids.

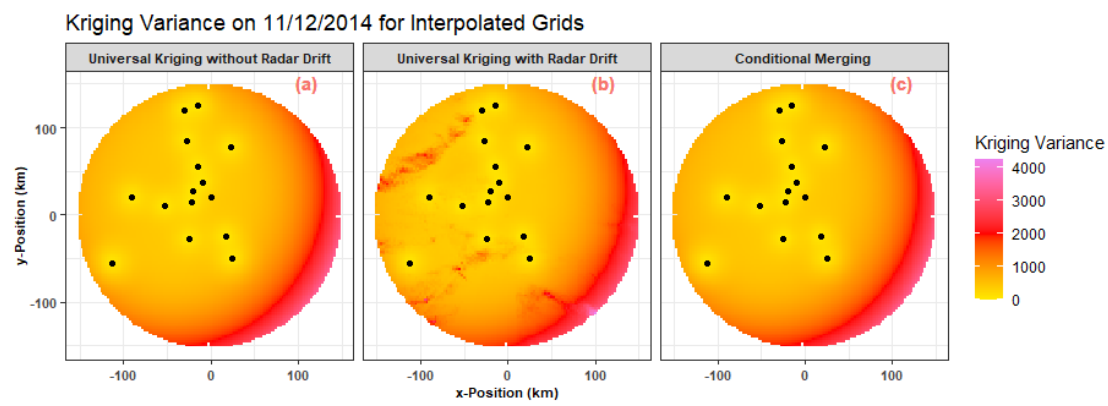

Figure 4. Plots of the Kriging variance for the three different interpolation methods on 11/12/2014. The interpolation methods are UK without radar drift (a), UK with radar drift (b) and conditional merging (c). 


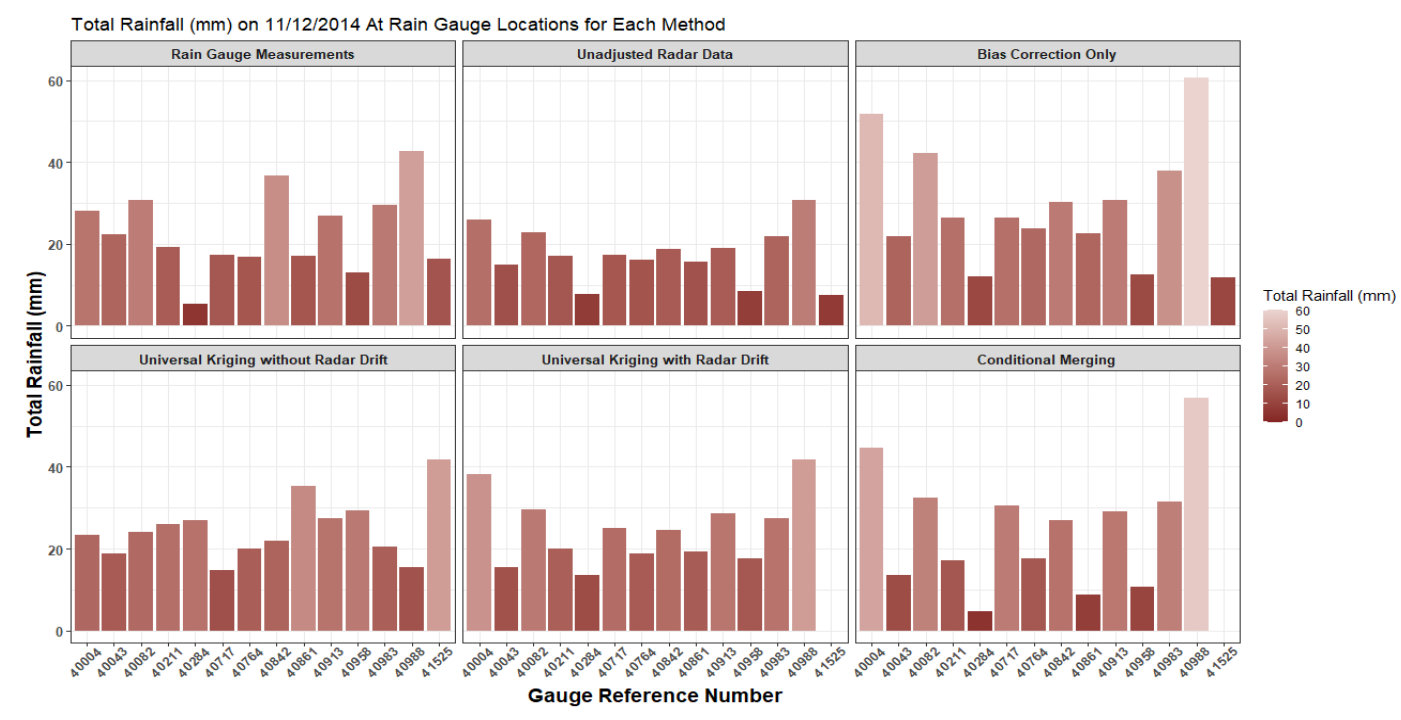

Figure 5. Rain gauge measurements and estimates for each method for 11/12/2014 taken from the rain gauge locations marked in Figure 3. One gauge reported NA for this day and was excluded from the plot.

\section{CONCLUSIONS AND RECOMMENDATIONS}

In this study we compared multiple approaches for generating gridded rainfall from radar and rain gauge data for a dataset with a limited number of rain gauges. For all approaches tested, we find a typical daily leave-one-out cross-validation RMSE on rainy days is between $10 \mathrm{~mm}$ and $20 \mathrm{~mm}$. We find that for Winter rainfall data all statistical merging approaches tested in this paper slightly improve accuracy over using unadjusted radar data on rainy days. These improvements were not observed for summer data indicating that a different approach to bias correction, such as correcting for bias on sub-daily data within individual rainfall events, could improve the results in future work. For the interpolation schemes, directional variograms could be produced to identify and model any anisotropy within the radar grid. The rainfall data could also be transformed to a gaussian distribution prior to interpolation to ensure the data is more suitable for Kriging. A comparison of radar data with a corresponding SILO grid demonstrated the potential for radar data to observe rainfall structures which might not be detected by rain gauges but without knowledge of the true rainfall it is difficult to assess the relative quality of the rainfall grids.

\section{REFERENCES}

Chumchean, S., Seed, A., Sharma, A., 2008. An operational approach for classifying storms in real-time radar rainfall estimation. Journal of Hydrology 363(1-4), 1-17.

Chumchean, S., Sharma, A., Seed, A., 2006. An integrated approach to error correction for real-time radarrainfall estimation. Journal of Atmospheric and Oceanic Technology 23(1), 67-79.

Cressie, N.A.C., 1993. Statistics for spatial data, 61pp. John Wiley \& Sons, New Jersey.

Jeffrey, S.J., Carter, J.O., Moodie, K.B., Beswick, A.R., 2001. Using spatial interpolation to construct a comprehensive archive of Australian climate data. Environmental Modelling \& Software 16(4), 309-330.

Jones, D.A., Wang, W., Fawcett, R., 2009. High-quality spatial climate data-sets for Australia. Australian Meteorological and Oceanographic Journal 58, 223-248.

Marshall, J.S., Langille, R.C., Palmer, W., Mc K., 1947. Measurement of rainfall by radar. Journal of the Atmospheric Sciences 4(6), 186-192.

Ochoa-Rodriguez, S., Wang, L.-P., Willems, P., Onof, C., 2019. A review of radar-rain gauge data merging methods and their potential for urban hydrological applications. Water Resources Research 55(8), 63566391.

Rabiei, E., Haberlandt, U., 2015. Applying bias correction for merging rain gauge and radar data. Journal of Hydrology 522, 544-557.

Seck, I., Baelen, J.V., 2018. Geostatistical merging of a single-polarized X-band weather radar and a sparse rain gauge network over an urban catchment. Atmosphere 9(12), 496. 